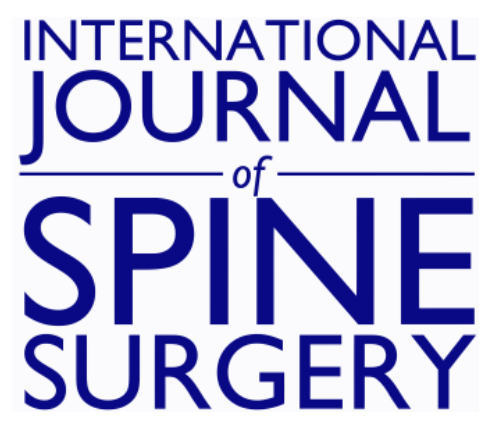

\title{
Clinical Application of a High Definition Three-Dimensional Exoscope in Anterior Lumbar Interbody Fusion: Technical Note
}

MANUELA D'ERCOLE, ELENA SERCHI, MATTEO ZANELLO, TOMMASO TUFO and CARMELO STURIALE

Int J Spine Surg 2020, 14 (6) 1003-1008

doi: https://doi.org/10.14444/7150

http://ijssurgery.com/content/14/6/1003

This information is current as of April 26, 2023.

Email Alerts Receive free email-alerts when new articles cite this article. Sign up at:

http://ijssurgery.com/alerts

The International Journal of Spine Surgery

2397 Waterbury Circle, Suite 1,

Aurora, IL 60504, Phone: +1-630-375-1432 


\title{
Clinical Application of a High Definition Three-Dimensional Exoscope in Anterior Lumbar Interbody Fusion: Technical Note
}

\author{
MANUELA D’ERCOLE, MD, PHD, ${ }^{1}$ ELENA SERCHI, MD,${ }^{2}$ MATTEO ZANELLO, MD,${ }^{3}$ TOMMASO TUFO, \\ MD, PHD ${ }^{4}$ CARMELO STURIALE, MD ${ }^{5}$ \\ ${ }^{I}$ Department of Neurosurgery, Fondazione Policlinico Universitario A. Gemelli Roma, Italy, ${ }^{2}$ Department of Neurosurgery, IRCCS Istituto delle Scienze \\ Neurologiche Bologna- Bellaria Hospital, Bologna Italy, ${ }^{3}$ Department of Surgery, Maggiore Hospital, Bologna Italy, ${ }^{4}$ Department of Neurosurgery, Fondazione \\ Policlinico Universitario A. Gemelli Roma, Italy, ${ }^{5}$ Department of Neurosurgery, IRCCS Istituto delle Scienze Neurologiche Bologna-Bellaria Hospital, Bologna \\ Italy
}

\begin{abstract}
Objectives: Video-assisted telescope operating monitor (VITOM) or exoscope is currently applied in different surgical specialties with clear visualization advantages in terms of magnification, illumination, and wide field of view. The small and deep surgical field of anterior lumbar interbody fusion (ALIF) seemed to be an ideal setting to assess efficacy of exoscope, also considering limits related to microscopic and endoscopic visualization currently employed.

Methods: We reported our preliminary experience with exoscope in 9 cases of ALIF at L5-S1 level. These data were retrospectively compared with those obtained from an equal sample of ALIF procedures performed with endoscope as visualization instrument. The technical aspects taken into account were time for procedure and blood loss. Reports from the surgeon about ergonomics and confidence with both techniques were also evaluated.

Results: Exoscope proved, in our experience, good visualization and ergonomics and unobstructed access to a small and deep surgical field, allowing abundant space to insert and manipulate the instruments.

Conclusions: The instrument contained dimension and its long working distance, superior to endoscope and comparable with operating microscope, showed clear advantages of maneuverability. Moreover, the stereoscopic vision provided by 3-dimensional images proved to be crucial in hand-eye coordination.
\end{abstract}

New Technology

Keywords: anterior lumbar interbody fusion, video-assisted telescope operating monitor, minimally invasive spine surgery, exoscopy

\section{INTRODUCTION}

Telescopic systems and cameras have been developed and refined over the past years, progressively improving the visualization and the illumination of the surgical field, thus allowing a wide and effective application of video-assisted telescope operating monitor (VITOM) or exoscope in many procedures within different surgical specialties.

In the present manuscript, the authors report their initial experience using the 3-dimensional (3D) exoscopic telescope as a visualization instrument in anterior lumbar interbody fusion (ALIF): in this application, the exoscope showed clear advantages about ergonomics and unobstructed access to a small and deep surgical field, offering an ideal visualization in terms of magnification, illumination, and wide field of view.

\section{MATERIALS AND METHODS}

Over a span time of 6 months, 9 ALIF procedures were performed at L5-S1 level at the neurosurgery department of Bellaria Hospital in Bologna, with the aid of high definition (HD) 3D exoscope as a visualization instrument. Patients were in supine position, under general anesthesia. Surgical exposure of anterior lumbosacral spine was performed by an abdominal surgeon, through a horizontal linear skin incision of 6 to $8 \mathrm{~cm}$ in length, in the lower abdominal quadrants and $5 \mathrm{~cm}$ under the navel (Figure 1). The rectus muscle and its anterior sheath were incised in their left paramedian side, approximately $1 \mathrm{~cm}$ far from the linea alba and then carefully retracted upward and laterally, preserving the inferior epigastric vessels, located on the inferior side of the muscle. 


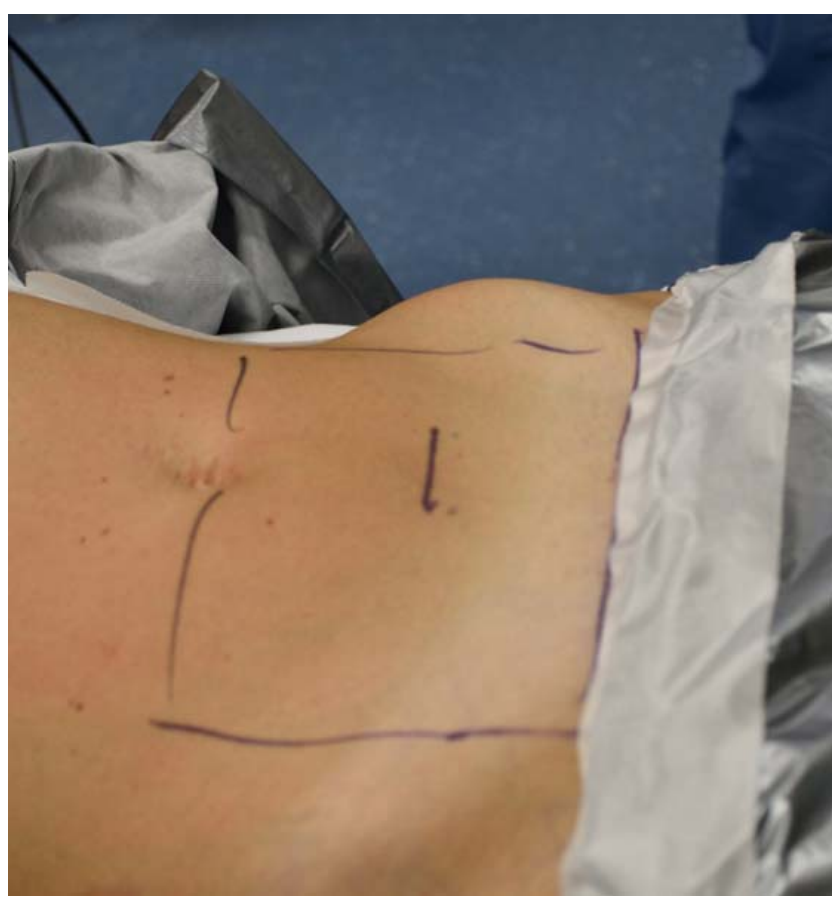

Figure 1. Surgical exposure of anterior lumbosacral spine is obtained through a skin incision in lower abdominal quadrants. Our preliminary experience is based on a linear horizontal skin incision of 6 to $8 \mathrm{~cm}$ in length, 5 to $7 \mathrm{~cm}$ beneath the umbilicus.

The arcuate line was then identified and cut in its most lateral portion, thus exposing the peritoneum and the left ureter, which were gently retracted medially, until the exposure of psoas muscle and big vessels was obtained. The L5-S1 disc space, our target, was identified between the bifurcation of aorta and cava into the 2 common iliac arteries and veins, respectively. After the complete exposure of the disc through retraction of each common iliac vessel on the respective side, deep retractors were inserted and fixed to the bony surface. Exoscope VITOM 3D (Karl Storz, Tuttlingen, Germany) was then inserted in the operating setting and maintained over the surgical field for the rest of the duration of the procedure. It consists of a specially developed telescope with zoom and focus functions, integrated illumination, and fiber optic light transmission. It is kept in position by an articulated, mobile, mechanical, holding arm (VESACRANE TM, Karl Storz Endoscopy, Tuttlingen, Germany). Through a control unit, the surgeon is usually able to focus, modify the magnification, and slightly shift the orientation of the scope. The surgical field was displayed on 2 movable 32" 3D monitors positioned on the opposite side of the surgical table respect to surgeon and assistant positions, which are usually

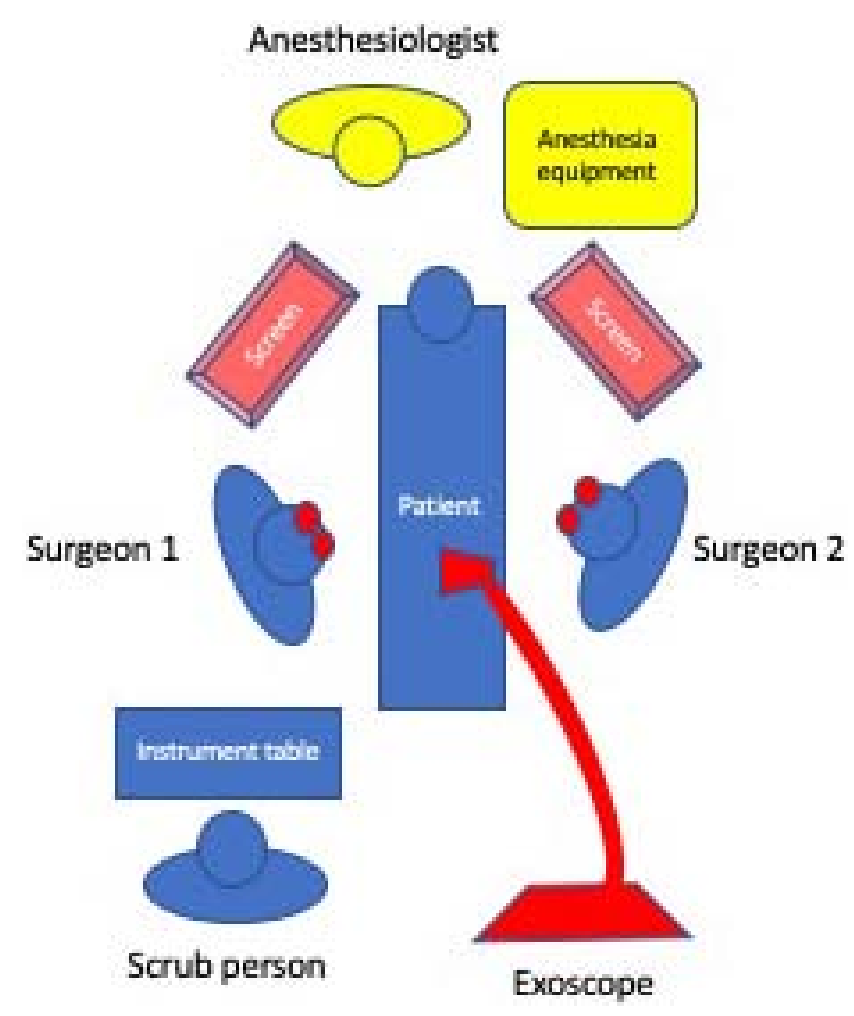

Figure 2. Exoscope video-assisted telescope operating monitor (VITOM) 3D in the operating setting, kept in position by an articulated holding arm, provides an unobstructed working space. The surgical field is displayed on 2 movable 32" 3D monitors positioned on the opposite side of the surgical table respect to surgeon and assistant positions, which are counterposed and wear 3D glasses. Images displayed on the 2 screens are specular to each other to reproduce the intraoperative sight with the correct orientation for each surgeon and ease surgical maneuvers. It is possible to shift from microscopic to macroscopic vision without moving the scope or completely losing microscopic vision, if necessary.

counterposed. A 1.5- to 2-m space is considered the closer available distance to avoiding blurry 3D images and allow the best clear visualization. Images displayed on the 2 screens are specular to each other to reproduce the intraoperative sight with the correct orientation for each surgeon and ease surgical maneuvers (Figure 2).

After incision of the anterior longitudinal ligament, a standard discectomy with careful curetting of the endplates was then completed (Figure 3). Finally, a tantalum cage filled by bone substitute, properly fitting the intervertebral space, was inserted and fixed with screws with an anterior plate under radioscopic control. After removal of the deep retractor and careful hemostasis, wound skin closure was completed. The technical aspects taken into account were time for procedure and blood loss (including amounts of blood collected from intraoperative suctioning and postoperative drainage, as inferred from the clinical charts). Reports from the 


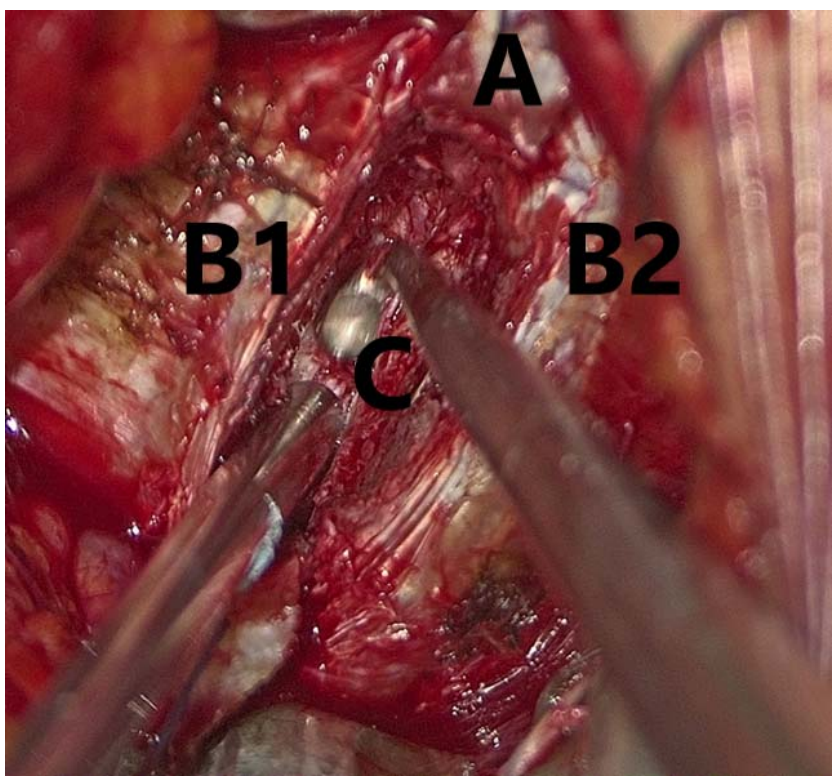

Figure 3. After incision and lateral retraction of anterior longitudinal ligament (A), discectomy and curetting of upper (B1) and lower (B2) endplates is performed under exoscopic magnification. The intervertebral space (C) is then prepared for insertion of the cage.

surgeon about ergonomics and confidence with exoscope were also evaluated. These data were retrospectively compared with those obtained from an equal sample of ALIF procedures performed according to the previous technique including endoscope as visualization instrument $(n=9)$.

\section{RESULTS}

The surgical procedure was performed in 9 patients affected by degenerative L5-S1 discopathy, complaining of low back pain not responsive to conservative treatments; 7 of them also reported radicular pain exacerbated by orthostatism; 1 of them had a previous interlaminar approach for herniectomy. Intraoperative x-rays and postoperative computed tomography scan showed satisfactory radiological results, consisting of segmentary restoration of physiological lordosis and widening of neural foramina (Figure 4). All patients reported satisfactory lumbar and radicular pain relief; no general and instrument-related complications were observed. Postoperative courses were uneventful with no significant pain, allowing early patient mobilization, and a brief hospitalization with discharge between 2 and 3 days after surgery. In surgeon personal experience, the adaptation to the exoscope was quite easy and immediate, requiring a gentle and shorter learning curve in comparison with the training in endoscopic technique.

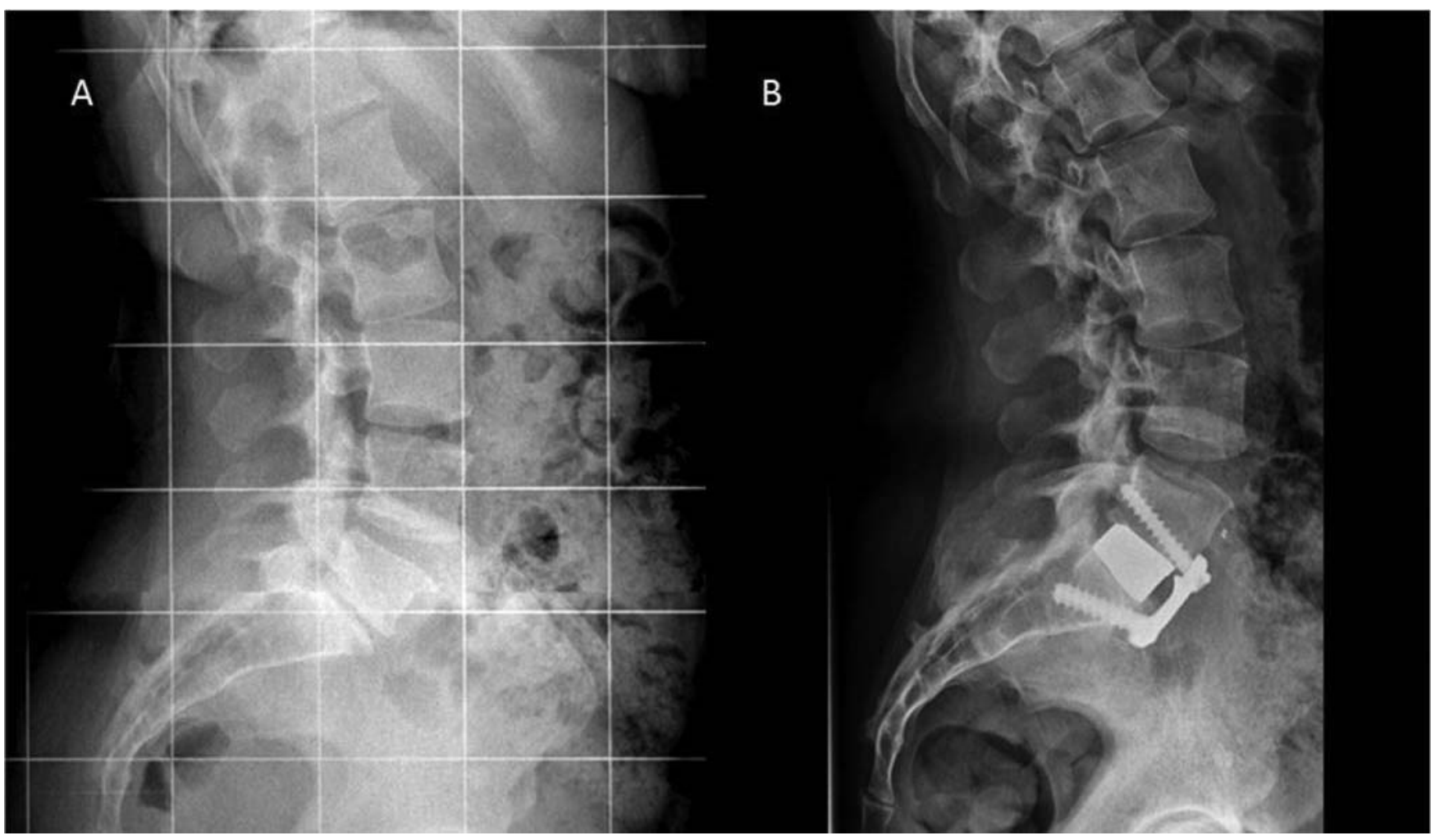

Figure 4. L5-S1 anterior lumbar interbody fusion (ALIF): X-rays presurgery (A) and postsurgery (B). Anterior access to the intervertebral space allows an easier insertion of angulated cages, when compared with posterior approaches, thus generating a satisfactory restoration of lumbar lordosis and spino-pelvic balance. Moreover, the restoration of disc height obtains increase of neuroforaminal volume and consequent relief of radicular symptoms due to indirect decompression. 


\section{DISCUSSION}

Telescopic systems and cameras consist of scopes with high magnification capacity and a large depth of field positioned over the surgical field, supported by a mobile mechanical arm and projecting to a screen. Developed and refined over the past decades, they evolved from former standard definition cameras and 2D visualization systems toward HD cameras and 3D instrumentations, allowing a wide and effective application of a new technology called VITOM or exoscope in many procedures within different surgical specialties. ${ }^{1-4}$ Due to its ergonomics and optimal vision, the application of exoscope in neurosurgery is progressively growing in popularity and gaining its role along with operating microscope and endoscopy, also thanks to the HD and $3 \mathrm{D}$ cameras, introduced to overcome the major drawbacks related to first prototypes such as lack of stereopsis. ${ }^{5}$ Surgical treatment of cranial tumors has been successfully performed with satisfactory results. ${ }^{6-11}$ Even in spinal surgery, many applications of exoscope have been reported, ${ }^{12}$ ranging between closure of lumbosacral myelomeningocele in a neonate $^{6}$ and treatment of degenerative disease. ${ }^{13}$ The small and deep surgical field of ALIF was chosen as a challenging setting to assess efficacy and advantages powered by VITOM.

Since its first application in the lumbar laparoscopic discectomy in $1991,{ }^{14}$ the anterior approach to the lumbar spine progressively grew up in popularity in the treatment of degenerative, tumoral, and infective diseases or even in revision surgery in case of nonunion after a previous posterior approach. ALIF shows similar fusion rates when compared with posterior lumbar interbody fusion (PLIF) and transforaminal lumbar interbody fusion (TLIF). ${ }^{15}$ Moreover, the anterior access to the intervertebral space usually allows an easier insertion of angulated cages, when compared with posterior approaches, thus generating a satisfactory restoration of lumbar lordosis and spino-pelvic balance. Restoration of disc height with consequent increase of neuroforaminal volume allows the relief of radicular symptoms due to radicular indirect decompression. ${ }^{15}$ The success of anterior approaches is mainly due to their minimal invasiveness, related to decreased blood loss and soft tissue injury when compared with standard posterior approaches, translating into less postoperative pain and recovery length. ${ }^{16}$ Also, in our experience, both postoperative pain and length of hospitalization were significantly lower when compared with posterior analogues arthrodesis procedures such as PLIF and TLIF, although a quantitative analysis of these data has not been performed, being beyond the purposes of this work. Surgical exposure of anterior lumbosacral spine is obtained through a skin incision in lower abdominal quadrants. Our preliminary experience is based on a linear horizontal skin incision of 6 to $8 \mathrm{~cm}$ in length, 5 to $7 \mathrm{~cm}$ beneath the umbilicus, thus allowing a wider and easier exposure of abdominal structures. Bassani et $\mathrm{al}^{17}$ proposed a very minimally invasive skin incision, "semilunar" shaped in the inferior aspect of the umbilicus; this technique was applied in a large series of patients with optimal aesthetical results.

In such a small and deep surgical field, the ideal visualization should provide a wide view, with magnification, illumination, ergonomics, and unobstructed access to the surgical set. The use of operating microscope is associated with several limitations mainly due to its large size, obstructing the access to surgical field and thus restricting the application of surgical instruments due to their length and their axis of movement, parallel to direction of view. Moreover, the binocular lenses of the operating microscope are limited in their movability, thus requiring uncomfortable and difficult positions to visualize structures in remote corners. Many authors proposed the employ of endoscopic-assisted visualization to overcome these difficulties. ${ }^{17}$ However, endoscopic view is characterized by the lack of $3 \mathrm{D}$ visualization unless the use of 3D endoscope, poor image quality compared with the operating microscope, and the frequent loss of visualization due to opacity related to evaporation or in case of significant intraoperative bleeding. Moreover, the endoscope needs to be held by one of the surgeons, thus subtracting "hands" for surgical maneuvers.

Exoscope or VITOM has been introduced as an alternative, allowing in our experience to overcome these difficulties. To the best of our knowledge, this is the first report concerning ALIF with VITOM 3D as a visualization instrument. No significant differences in time for procedure and blood loss were noticed in our retrospective comparison with endoscopic-assisted technique. Advantages of exoscopic telescope over microscope are mainly related to its longer focal distance and wider field of view. It is not bulky and can be positioned approximately 
$200 \mathrm{~mm}$ or even farther above the surgical field, so providing an unobstructed working space allowing an easy introduction and passage of surgical instruments. Both surgeon and his assistant can work in a natural and comfortable ergonomic position: from their counterposed position, they can visualize the surgical field through two 32" 3Dmonitors positioned on the opposite side of the surgical table respect to each one, reproducing exactly the intraoperative sight with the correct orientation to each one, thus easing surgical maneuvers. Moreover, it is possible to shift from microscopic to macroscopic vision in a rapid and easy way, without moving the scope or completely losing microscopic vision, if necessary. In our experience, the adaptation to the exoscope was quite easy, probably because of our previous experience with endoscopy in cranial and spinal surgery. This evidence was also reported by Beez et $\mathrm{al}^{6}$ in description of learning curve in exoscopy in pediatric neurosurgeons used to endoscopic surgery, because of similarities between these 2 working environments, such as watching the operating theatre on a high-definition $3 \mathrm{D}$ screen. As previously reported, the main drawback is cumbersomeness in repositioning and refocusing, referable to the mechanical holding arm. As already outlined, this aspect could probably be improved by applying a hydraulic counterbalance or a robotic mechanism to the holding arm.

\section{CONCLUSIONS}

In our experience, exoscopy is a useful and efficient tool in the anterior approach to lumbar spine. Notably, the instrument contained dimension and its long working distance, superior to endoscope and comparable with operating microscope, showed clear advantages of maneuverability, allowing abundant space to insert and manipulate the instruments. Other advantages were regarded as the wide field of view and deep focus, thus minimizing the need for repositioning and refocusing during the procedure. In such a deep surgical field, the stereoscopic vision provided by $3 \mathrm{D}$ images seems to be crucial in hand eye coordination.

\section{REFERENCES}

1. Frykman PK, Duel BP, Gangi A, et al. Evaluation of a video telescopic operating microscope (VITOM) for pediatric surgery and urology: a preliminary report. J Laparoendosc $A d v$ Surg Tech A. 2013;23(7):639-643.
2. Krishnan KG, Schöller K, Uhl E. Application of a compact high-definition exoscope for illumination and magnification in high-precision surgical procedures. World Neurosurg. 2017;97:652-660.

3. Mamelak AN, Danielpour M, Black KL, et al. A highdefinition exoscope system for neurosurgery and other microsurgical disciplines: preliminary report. Surg Innov. 2008;15(1):38-46.

4. Vercellino GF, Erdemoglu E, Kyeyamwa S, et al. Evaluation of the VITOM in digital high-definition video exocolposcopy. J Low Genit Tract Dis. 2011;15(4):292-295.

5. Parihar V, Yadav YR, Kher Y, et al. Learning neuroendoscopy with an exoscope system (video telescopic operating monitor): early clinical results. Asian $J$ Neurosurg. 2016;11(4):421-426.

6. Beez T, Munoz-Bendix C, Beseoglu K, et al. First clinical applications of a high definition three-dimensional exoscope in pediatric neurosurgery. Cureus. 2018 Jan 24;10(1):e2108. doi: 10.7759/cureus.2108.

7. Birch K, Drazin D, Black KL, et al. Clinical experience with a high definition exoscope system for surgery of pineal region lesions. J Clin Neurosci. 2014;21(7):1245-1259.

8. Mamelak AN, Drazin D, Shirzadi A, et al. Infratentorial supracerebellar resection of a pineal tumor using a high definition video exoscope (VITOM ${ }^{\circledR}$ ). J Clin Neurosci. 2012;19(2):306-309.

9. Mamelak AN, Nobuto $\mathrm{T}$, Berci G. Initial clinical experience with a high-definition exoscope system for microneurosurgery. Neurosurgery. 2010;67(2):476-483.

10. Piquer J, Llácer JL, Rovira V, et al. Fluorescence-guided surgery and biopsy in gliomas with an exoscope system. Biomed Res Int. 2014;2014:207974. doi: 10.1155/2014/207974

11. Rossini Z, Cardia A, Milani D, et al. Video Telescope Operating Monitor (VITOM) 3D: preliminary experience in cranial surgery. Technical case report. World Neurosurg. 2017;107:663-668.

12. Moisi MD, Hoang K, Tubbs RS, et al. Advancement of surgical visualization methods: comparison study between traditional microscopic surgery and a novel robotic optoelectronic visualization tool for spinal surgery. World Neurosurg. 2017;98:273-277.

13. Shirzadi A, Mukherjee D, Drazin DG, et al. Use of the video telescope operating monitor (VITOM) as an alternative to the operating microscope in spine surgery. Spine (Phila Pa 1976). 2012;37(24):E1517-E1523.

14. Obenchain TG. Laparoscopic lumbar discectomy: case report. J Laparoendosc Surg. 1991;1(3):145-149.

15. Teng I, Han J, Phan K, et al. A meta-analysis comparing ALIF, PLIF, TLIF and LLIF. J Clin Neurosci. 2017;44:11-17.

16. Mobbs RJ, Phan K, Malham G, et al. Lumbar interbody fusion: techniques, indications and comparison of interbody fusion options including PLIF, TLIF, MI-TLIF, OLIF/ATP, LLIF and ALIF. $J$ Spine Surg. 2015;1(1):2-18.

17. Bassani R, Querenghi AM, Cecchinato R, et al. A new "keyhole" approach for multilevel anterior lumbar interbody fusion: the perinavel approach-technical note and literature review. Eur Spine J. 2018;27(8):1956-1963.

Disclosures and COI: The Authors declare that there is no conflict of interest with respect to the 
research, authorship, and/or publication of this article. The authors received no financial support for the research, authorship, and/or publication of this article.

Corresponding Author: Manuela D'Ercole, MD, PhD, Department of Neurosurgery, Fondazione Policlinico Universitario A. Gemelli Roma, Largo Agostino Gemelli 8, 00168 Rome Italy.
Phone: +393208674589; Email: manuela.dercole@ policlinicogemelli.it.

Published 22 January 2021

This manuscript is generously published free of charge by ISASS, the International Society for the Advancement of Spine Surgery. Copyright (C) 2020 ISASS. To see more or order reprints or permissions, see http://ijssurgery.com. 